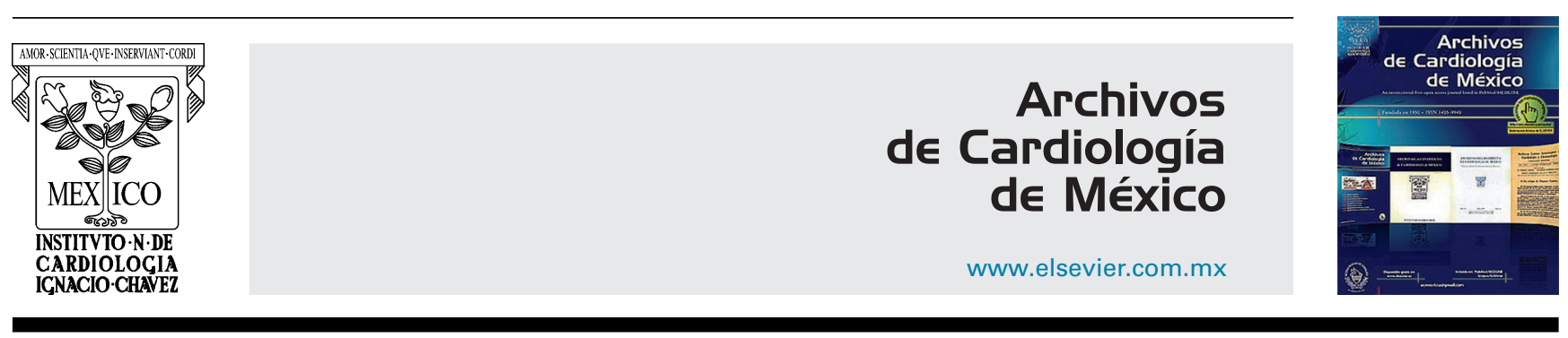

CARTA AL EDITOR

\section{Masa tumoral cardiaca en un paciente con linfoma de Burkitt. Caso clínico}

\section{Cardiac tumor mass in a patient with Burkitt's lymphoma. Clinical case}

El compromiso cardiaco en el linfoma de Burkitt es rara vez descrito en la literatura ${ }^{1}$. Afecta con mayor frecuencia a la aurícula derecha (74\%), al ventrículo derecho (14\%) y a la aurícula izquierda, venas pulmonares y pericardio (12\%).

La ecocardiografía transtorácica tiene una sensibilidad cercana al $100 \%^{2}$, y juega un rol importante no solo en el diagnóstico, sino también en el tratamiento y seguimiento de estos pacientes ${ }^{3}$.

El objetivo de este estudio fue describir la utilidad de la ecocardiografía en el diagnóstico y seguimiento de masas intracardiacas en un niño de 5 años con linfoma de Burkitt.

Paciente varón de 5 años de edad, sin antecedentes de importancia; con tiempo de enfermedad de 2 meses, caracterizado por dolor abdominal, ictericia y coluria. Debido a la persistencia de los síntomas descritos, acude al hospital general donde se evidencia anemia, trombocitopenia, hepatomegalia con leve dilatación de vías biliares, lesión sólida contigua al riñón izquierdo que se extiende al flanco izquierdo, y por ecocardiografía una imagen tumoral que abarca casi la totalidad de la aurícula derecha con protrusión a través de la válvula tricúspide hacía el ventrículo derecho, por lo que es hospitalizado en el Instituto Nacional de Enfermedades Neoplásicas con el diagnóstico de síndrome linfoproliferativo primario retroperitoneal y tumor intracardiaco.

A su ingreso se encontró taquicárdico (FC: $100 \mathrm{~min}$ ), taquipnéico (FR: $28 \mathrm{~min})$ e hipotenso $(80 / 40 \mathrm{mmHg})$. El paciente estaba adelgazado, pálido, ictérico con presencia de adenopatías de $\pm 0,5 \mathrm{~cm}$ cervicales, axilares e inguinales. Ruidos cardiacos rítmicos de baja tonalidad, campos pulmónares con murmullo vesicular normal, no ingurgitación yugular. Abdomen globuloso con hepatomegalia $(10 \mathrm{~cm}$ por debajo del reborde costal derecho) y esplenomegalia. Testículos normales, pero con edema escrotal.

Los exámenes de laboratorio del 20/08/2015 mostraron: hemoglobina $6,2 \mathrm{~g} / \mathrm{l}$; hematocrito $20,2 \%$; leucocitos $17,97 \times 10^{9} /$; linfocitos $14 \%$; monocitos $2 \%$; basófilos $1 \%$; segmentados $83 \%$; plaquetas $547 \times 10^{9} /$; INR 1,07 ; glucosa $4 \mathrm{mmol} / \mathrm{l}$; creatinina $30 \mu \mathrm{mol} / \mathrm{l}$; proteínas totales $51 \mathrm{~g} / \mathrm{l}$; albúmina 24,6 g/l; globulina $26 \mathrm{~g} / \mathrm{l}$; bilirrubina total $164,7 \mu \mathrm{mol} / \mathrm{l}$; bilirrubina directa $150 \mu \mathrm{mol} / \mathrm{l}$; bilirrubina indirecta 13,8 $\mu \mathrm{mol} / \mathrm{l}$; fosfatasa alcalina $3.175 \mathrm{U} / \mathrm{l}$; TGP $365 \mathrm{U} / \mathrm{l} ;$ TGO 575U/l; G-glutamiltranspeptidasa $891 \mathrm{U} / \mathrm{l}$; DHL $3.803 \mathrm{U} / \mathrm{l}$; electrolitos normales y perfil viral para hepatitis $B, C$, HIV y HTLV1 negativo.

La biopsia de la médula ósea $(21 / 08 / 2015)$ con áreas hipoplásicas y zonas hiperplásicas, sin células neoplásicas y la biopsia de ganglio de axila derecha demostró linfoma no Hodgkin. La gammagrafía ósea resultó negativa.

En la evaluación cardiológica, el paciente se encontraba asintomático cardiovascular, con signos vitales estables y examen físico cardiológico normal. El electrocardiograma en ritmo sinusal con frecuencia $71 \mathrm{lpm}$, trastornos de la conducción del ventrículo derecho y alteraciones difusas de la repolarización ventricular. La ecocardiografía transtorácica mostró 3 masas intracardiacas en la aurícula derecha de $3,8 \times 3,3 \times 2,5$ y $2 \times 2 \mathrm{~cm}$, respectivamente; adosadas al tabique interauricular, con protrusión durante la diástole a través de la válvula tricúspide (fig. 1A), siendo considerado como progresión tumoral, se hizo el diagnóstico diferencial con trombo vs. endocarditis infecciosa. La función ventricular normal con fracción de expulsión del 70\% y el pericardio de características normales.

El 25 de agosto de 2015 ingresa en la unidad de cuidados intensivos $(\mathrm{UCl})$ para inicio del tratamiento con quimioterapia pre-fase: hidratación, alcalinización, dexametasona, vincristina y ciclofosfamida, con manejo de cuadro de lisis tumoral; siendo dado de alta de la UCl el 30/08/2015. El ecocardiograma de control mostró una reducción de la masa cardiaca en un $80 \%$ (fig. 1B).

Continua con quimioterapia del esquema de tratamiento, y del 3 al 7 de septiembre recibió quimioterapia bloque A: dexametasona $10 \mathrm{mg} / \mathrm{m}^{2} / \mathrm{d} / 5 \mathrm{~d}$; vincristina $1,5 \mathrm{mg} / \mathrm{m}^{2} / \mathrm{d}$; ifosfamida $800 \mathrm{mg} / \mathrm{m}^{2} / \mathrm{d} / 5 \mathrm{~d}$; metotrexato $2.000 \mathrm{mg} / \mathrm{m}^{2} / \mathrm{d}$; citarabina $150 \mathrm{mg} / \mathrm{m}^{2} / 12 \mathrm{~h} / 2 \mathrm{~d}$ y etopósido $100 \mathrm{mg} / \mathrm{m}^{2} / \mathrm{d} / 2 \mathrm{~d}$. No presentó intercurrencias.

Anatomía patológica (9/09/2015): linfoma B de alto grado con inmunofenotipo compatible con linfoma de Burkitt:

$$
\mathrm{CD} 20(+) \rightarrow \mathrm{CD} 3(-) \rightarrow \mathrm{Ki} 67 \text { 100\% }
$$

$\mathrm{CD} 79 \mathrm{a}(+) \rightarrow$ TdeT $(-)$ 

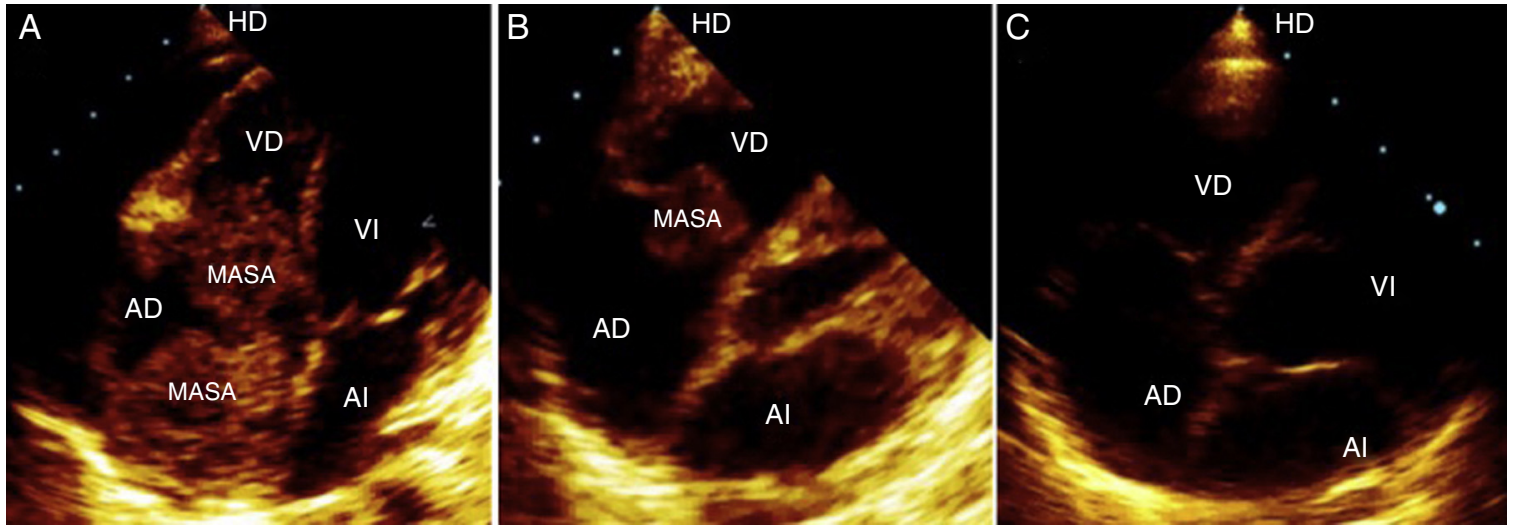

Figura 1 A) Imagen ecocardiográfica, en el plano apical de 4 cámaras, que muestra 3 masas en aurícula derecha que protruyen al ventrículo derecho durante la diástole, antes del tratamiento (25/08/2015). B) Después del tratamiento con quimioterapia pre-fase se observa una reducción del $80 \%$ de las masas tumorales, $(01 / 09 / 2015)$. C) Después del tratamiento con quimioterapia bloque A y bloque $B$, hay una remisión completa de las masas tumorales.

$\mathrm{CD} 10(+) \rightarrow \mathrm{BCL} 2(-)$

$\mathrm{BCL6}(+) \rightarrow \mathrm{MPO}(-)$

Se hospitaliza para administración de quimioterapia bloque B: (del 22 al 26 de octubre) dexametasona $10 \mathrm{mg} / \mathrm{m}^{2} / \mathrm{d} / 5 \mathrm{~d}$; vincristina $1,5 \mathrm{mg} / \mathrm{m}^{2} / \mathrm{d}$; ciclofosfamida $200 \mathrm{mg} / \mathrm{m}^{2} / \mathrm{d} / 5 \mathrm{~d}$; metotrexato $2.000 \mathrm{mg} / \mathrm{m}^{2} / \mathrm{d}$ y doxorubicina $25 \mathrm{mg} / \mathrm{m}^{2} / 2 \mathrm{~d}$.

En el ecocardiograma del 28/10/2015 no se observa masa cardiaca (fig. 1C), y la función ventricular es normal. El paciente tuvo una evolución clínica favorable. Actualmente continua en quimioterapia hasta completar 3 ciclos $\mathrm{A}$ y 3 ciclos B.

La presencia de tumores en el corazón es rara, con una incidencia del $0,05 \%^{4}$, siendo los mixomas los más frecuentes, con una frecuencia del $50 \%$, los cuales son de una estirpe benigna; mientras que los sarcomas con mucha menor frecuencia (20\%) caracterizan a los tumores malignos (Barnes en 1934 diagnostica el primer sarcoma primario del corazón $)^{5}$. La relación entre tumores benignos/malignos es del orden de 3/1. El hallazgo de linfoma representa entre el 1-2\% de todos los tumores primarios del corazón, y se presenta con mayor frecuencia en sujetos inmunosuprimidos ${ }^{6}$. Hay 2 presentaciones de linfoma de Burkitt, el endémico y el esporádico. El primero es el cáncer más común en Africa ${ }^{7}$, comprometiendo la mandíbula y las vísceras abdominales, mientras que el segundo es raro, pero de mayor agresividad, afectando la región ileocecal y el peritoneo. El linfoma de Burkitt afecta a pacientes desde los 4 a 20 años, siendo más común en varones. En la mayoría de los casos los niños son sanos y activos, asintomáticos previo a la enfermedad. El cuadro clínico es muy variable y depende del tamaño de la masa, su ubicación y de la repercusión hemodinámica. Generalmente cuando la ubicación es en cavidades izquierdas, el paciente refiere disnea, palpitaciones, dolor torácico, mientras que cuando involucra cavidades derechas se manifiesta por fatiga, distensión abdominal y edema de miembros inferiores. Con frecuencia se asocia a derrames pericárdicos que pueden evolucionar tórpidamente hasta producir un taponamiento cardiaco ${ }^{8}$.
El tratamiento quirúrgico de un linfoma cardiaco no es la mejor alternativa, debido a que la resección del tumor es difícil y generalmente incompleto ${ }^{9}$. La quimioterapia secuencial y alternada debe ser considerada como la mejor alternativa terapéutica, la remisión completa ha sido documentada en menos del $60 \%$ de los $\operatorname{casos}^{10}$.

La ecocardiografía juega un rol importante, sobre todo para su identificación temprana y abordaje oportuno, así como para su seguimiento. En este caso las masas tumorales no generaron sintomatología, y la quimioterapia permitió la resolución completa del linfoma de Burkitt cardiaco.

\section{Financiación}

No se recibió patrocinio de ningún tipo para llevar a cabo este artículo.

\section{Bibliografía}

1. Meshref M, Sassolas F, Schell M, et al. Primary cardiac Burkitt lymphoma in a child. Pediatr Blood Cancer. 2004;42: 380-3.

2. Fresneau B, Oberlin $O$, Brugieres $L$, et al. Les tumeurs cardiaques primitives malignes de l'enfant et de l'adolescent. Arch Pédiatrie. 2010;17:495-501.

3. Desgranges Z, Deubner H, Broudy V, et al. Burkitt lymphoma of the heart. Echocardiographically documented improvement with chemotherapy. J Diag Med Sonography. 2011;27:186-90.

4. Tzachanis D, Dewar R, Luptakova K, et al. Primary cardiac Burkitt lymphoma presenting with abdominal pain. Case Rep Hematol. 2014;2014:687598.

5. Silverman N. Primary cardiac tumors. Ann Surg. 1980;191: 127-38.

6. Alcocer Gamba MA, León González S, Castro Montes E, et al. Diagnóstico de linfoma no Hodgkin con infiltración intraauricular. Invest Clin. 2012;53:289-300.

7. Walusansa V, Okuku F, Orem J. Burkitt lymphoma in Uganda, the legacy of Denis Burkitt and an update on the disease status. $\mathrm{Br}$ J Hematol. 2012;156:757-60.

8. Miguel C, Bestetti R. Primary cardiac lymphoma. Intenational J Cardiol. 2011;149:358-63. 
9. Legault S, Couture C, Bourgault C, et al. Primary cardiac Burkitt-like lymphoma of the right atrium. Can J Cardiol. 2009;25:163-5.

10. Fuzellier JF, Saade YA, Torossian PF, et al. Primary cardiac lymphoma: Diagnosis and treatment. Report of 6 cases and review of the literature. Arch Mal Coeur Vaiss. 2005;98:875-80.

Enrique Ruiz-Mori ${ }^{a, *}$, Leonor Ayala-Bustamantea ${ }^{\mathrm{b}}$, Hernán Ruiz-Morib ${ }^{c}$, Jorge Burgos-Bustamante ${ }^{a}$, Clara Pérez-Samitier ${ }^{\mathrm{C}}$ y Mayer Zaharia-Bassan ${ }^{\mathrm{d}}$

a Servicio de Cardiología, Instituto Nacional de Enfermedades Neoplásicas, Lima, Perú b Departamento de Ayuda al Diagnóstico, Hospital de Emergencias Pediátricas, Lima, Perú

c Departamento de Oncología Pediátrica, Instituto Nacional de Enfermedades Neoplásicas, Lima, Perú

d Departamento de Radioterapia, Instituto Nacional de Enfermedades Neoplásicas, Lima, Perú

* Autor para correspondencia. Instituto Nacional de Enfermedades Neoplásicas, Av. Angamos Este 2520, Surquillo 15038, Lima, Perú. Teléfono: +51-1-4213208. Correo electrónico: cruizm@usmp.pe (E. Ruiz-Mori). 